\title{
Cluster Assembly in Hierarchically Collapsing Clouds
}

\author{
Enrique Vázquez-Semadeni ${ }^{1}$, Alejandro González-Samaniego ${ }^{2}$, \\ Manuel Zamora-Avilés ${ }^{1}$ and Pedro Colín ${ }^{1}$ \\ ${ }^{1}$ Instituto de Radioastronomía y Astrofísica, UNAM \\ Antigua Carretera a Pátzcuaro \# 8701, Morelia, Michoacán, 58088, México \\ email: e.vazquez,m.zamora,p.colin@crya. unam.mx \\ ${ }^{2}$ Instituto de Astronomía, UNAM, \\ Circuito Exterior, S/N, México, D.F., 04510, México \\ email: ags@astro.unam.mx
}

\begin{abstract}
We discuss the mechanism of cluster formation in hierarchically collapsing molecular clouds. Recent evidence, both observational and numerical, suggests that molecular clouds (MCs) may be undergoing global, hierarchical gravitational collapse. The "hierarchical" regime consists of small-scale collapses within larger-scale ones. The latter implies that the star formation rate increases systematically during the early stages of evolution, and occurs via filamentary flows onto "hubs" of higher density, mass, and velocity dispersion, and culminates a few Myr after than the small-scale collapses have started to form stars. In turn, the small-scale collapses occur in clumps embedded in the filaments, and are themselves falling into the larger potential well of the still-ongoing large-scale collapse. The stars formed in the early, small-scale collapses share the infall motion of their parent clumps towards the larger potential trough, so that the filaments feed both gaseous and stellar material to the hubs. This leads to the presence of older stars in a region where new protostars are still forming, to a scale-free or fractal structure of the clusters, in which each unit is composed of smaller-scale ones, and to the eventual merging of the subunits, explaining the observed structural features of open clusters.
\end{abstract}

Keywords. Galaxies: star clusters, Gravitation, Hydrodynamics, ISM: clouds, Stars: formation

\section{Introduction}

It is presently accepted that most stars form in clusters, although the details of the cluster-formation process, especially the origin of their structural properties, remain a matter of active research (e.g., Lada \& Lada 2003). In particular, a number of structural properties of the clusters have been uncovered that still require an adequate theoretical understanding, such as: i) the existence of a mass segregation in the clusters, with the most massive stars lying closer to the cluster's center (Hillenbrand \& Hartmann 1998); ii) the distribution of protostellar separations, which appears to have no characteristic scale (Bressert et al. 2010); iii) the likely existence of an age gradient in clusters, with the youngest stars being located in the highest-density regions (Kuhn et al. 2015a).

Numerical simulations have begun to offer some insight about these properties. Kirk et al. (2014) have concluded, from a suite of simulations of self-gravitating, decaying isothermal turbulence, that the most massive stars form in situ at the cluster centers, rather than "sinking" there through dynamical interactions in the cluster itself. However, they gave no physical explanation as to why the most massive stars should form there. More recently, Kuhn et al. (2015b) have suggested, by comparing multi-wavelength observations of stellar clusters with numerical simulations, that clusters form by mergers of "subcluster" structures, although again no explanation of why such mergers should 
occur is provided. The presence of subunits of somewhat different ages in the clusters has also been pointed out by Rivera-Gálvez et al. (2015).

A physical mechanism capable of providing a unifying scenario to these properties is the one advanced by Vázquez-Semadeni et al. (2009). These authors proposed that, if molecular clouds (MCs) are assembled by large-scale colliding streams of warm, atomic gas, they rapidly condense into the cold atomic phase, becoming Jeans-unstable and beginning to collapse globally. Moreover, the collision of the streams causes moderately supersonic turbulence (e.g., Koyama \& Inutsuka 2002; Heitsch et al. 2005) in the cold gas, which produces a spectrum of density fluctuations, where large-amplitude, smallscale (LASS) fluctuations are superposed on smaller-amplitude, larger-scale (SALS) ones (e.g., Kim \& Ryu 2005). Since these density fluctuations are nonlinear, the LASS fluctuations have shorter free-fall times than the SALS ones, therefore completing their collapse earlier. This process is therefore similar to Hoyle's (1953) fragmentation, except that the density fluctuations are of turbulent origin and are therefore nonlinear. In what follows, we will refer to this scenario as "hierarchical gravitational collapse" (HGC).

This scenario also predicts that the star formation rate (SFR) in molecular clouds (MCs) evolves over time, as a consequence of the evolution of the clouds themselves as they go through global gravitational collapse (Zamora-Avilés et al. 2012; Hartmann et al. 2012; Zamora-Avilés \& Vázquez-Semadeni 2014).

In this contribution, we describe results of a model on the evolution of the SFR and of cluster assembly in a numerical simulation of MC formation and evolution, emphasizing the role of $\mathrm{HGC}$ in the resulting structure of the cluster.

\section{A model for the evolution of the SFR in molecular clouds}

Several models for the SFR and the core mass function (CMF) have been put forward in the last decade (e.g., Krumholz \& McKee 2005; Hennebelle \& Chabrier 2011; Padoan \& Nordlund 2011), all based on the notion that MCs are globally supported by supersonic turbulence, and with the SFR given by the collapse of local density fluctuations induced by the turbulence.

Operationally, the computation of the SFR in these models is performed by using the probability density function (PDF) of the density field - which is known to have a lognormal form for a non-self-gravitating, isothermal turbulent gas (Vázquez-Semadeni 1994) to compute the mass fraction at densities above a suitably defined density threshold, and dividing it by a timescale characteristic of its density range. Details of the similarities and differences between these models have been given by Federrath \& Klessen (2012). Models of this kind are intrinsically stationary, as they assume that the MCs are in approximate virial equilibrium between turbulence and gravity. In these models, the main controlling parameters are those characterizing the turbulence - the Mach number $M_{\mathrm{s}}$, the virial parameter $\alpha$ (Bertoldi \&McKee 1992), and perhaps the ratio of compressible to solenoidal energy (Federrath \& Klessen 2012).

However, the basic premise of equilibrium in the stationary models is inconsistent with the scenario of HGC, since in the latter, the cloud is assumed to be in global gravitational collapse. A model combining the calculation of small-scale collapse as in the above models with the large-scale collapse of the whole cloud has been presented by Zamora-Avilés et al. (2012, hereafter Z+12) and Zamora-Avilés \& Vázquez-Semadeni (2014, hereafter ZV14). In what follows we will refer to this as "the evolutionary model". In it, in addition to the calculation of the instantaneous SFR as in the models mentioned above, the evolution and collapse of the cloud (with the associated systematic increase of its mean density) are also followed in time. The cloud is assumed to be formed by the collision of warm atomic 

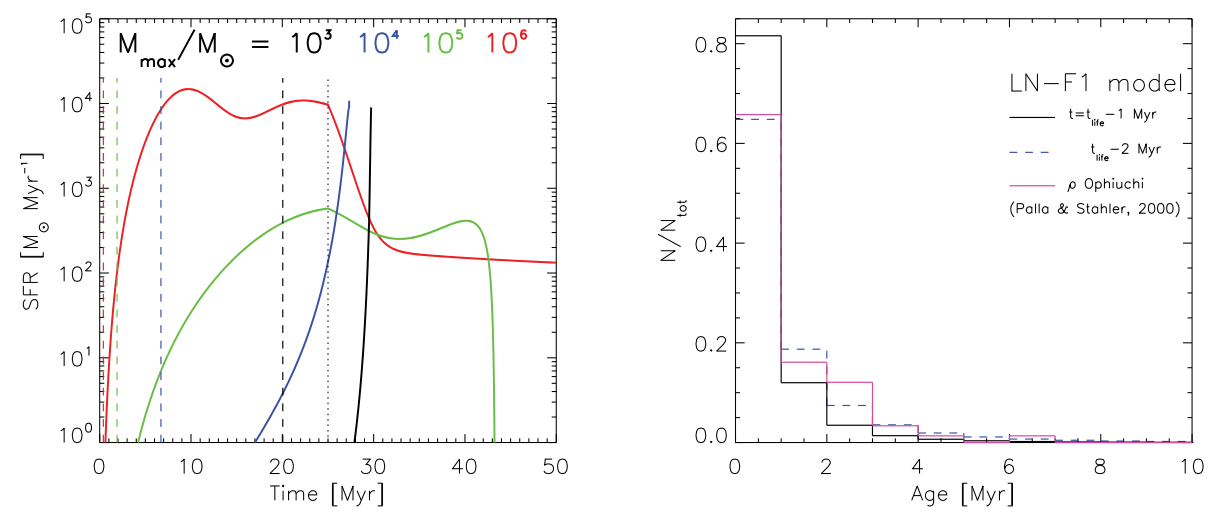

Figure 1. Left: Time evolution of the SFR for clouds with $M_{\max }=10^{3}, 10^{4}, 10^{5}$, and $10^{6} \mathrm{M}_{\odot}$ (black, blue, green and red lines respectively). The vertical dashed lines represent the time at which the clouds start to form stars. The vertical black dotted line represents the time at which accretion onto the clouds from the warm medium stops (from ZV14). Right: Normalized stellar age histograms for a model cloud at 1 (solid black line) and 2 (dashed blue line) Myr before the cloud is destroyed by the ionizing stellar feedback. Also shown is the corresponding histogram for the embedded cluster in the $\rho$-Oph cloud (dash-dot-dot magenta line; Palla \& Stahler 2000) (from $\mathrm{Z}+12$.$) .$

streams, and to grow in mass by the continued accretion of this material, which undergoes a phase transition to the cold phase as it enters the cloud. The cloud also develops turbulence through a combination of the nonlinear thin-shell instability (Vishniac 1994) and the Kelvin-Helmholz, Rayleigh-Taylor, and thermal instabilities (see, e.g., Heitsch et al. 2005). Because the cloud is collapsing, the instantaneous mass fraction at high densities increases in time, and therefore so does the instantaneous SFR. Knowing the SFR at all times, the total mass in stars can be computed and, assuming a standard IMF, the instantaneous number of massive stars is also computed. These feed back on the cloud through ionizing UV radiation, and the rate of ionization can be computed using standard prescriptions (Franco et al. 1994), eventually destroying the cloud.

Contrary to the stationary equilibrium models, in the evolutionary model the turbulence parameters are not free, but rather they are assumed to have the (fixed) values typical of the cold atomic medium. Thus, the turbulence is assumed to be moderately supersonic, with Mach number $M_{\mathrm{s}} \sim 3$ (e.g., Heiles \& Troland 2003). The only remaining free parameter is the total mass involved in the cloud-formation and evolution process $\left(M_{\max }\right)$. Figure 1 shows, in the left panel, the evolution of the SFR for clouds of various masses according to the model. In the right panel, this figure shows the histogram of stellar ages in a cluster that evolves according to the evolution of the SFR predicted by the model in a 2000-M $\odot$ cloud, 1 and 2 Myr before the cloud is destroyed by the ionizing stellar feedback. The figure also shows the normalized stellar age histogram in the $\rho$-Ophiucus cloud (Palla \& Stahler 2000), showing that the model at 2 Myr before destruction matches quite closely the observed histogram. The match is expected to occur some time before destruction because the clusters studied by Palla \& Stahler (2000) are still embedded in their parent clouds, suggesting that the destruction of the clouds is not completed yet.

\section{A simulation of cluster formation}

The results from the model described in the previous section are supplemented by those obtained in a numerical simulation of cloud formation, evolution and destruction, 

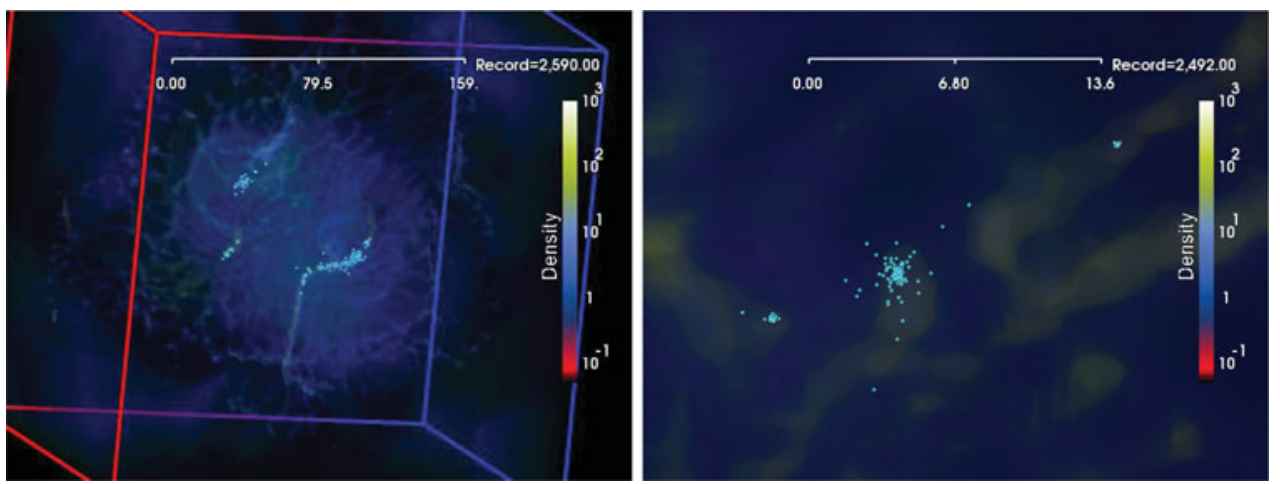

Figure 2. Two views of the numerical simulation. Left panel: A global projected view at $t=25.9 \mathrm{Myr}$, showing the three clusters that form within filamentary structures. The box size is $256 \mathrm{pc}$ and the ruler shows a scale of $159 \mathrm{pc}$. The color bar indicates the local density in $\mathrm{cm}^{-3}$. Right panel: A zoom-in around the intermediate-mass cluster at $t=24.9 \mathrm{Myr}$, showing that it is composed of three subunits at this time, which later $(t \sim 30$ Myr) merge to form a single larger cluster. The central object in this image is the cluster whose evolution is shown in Fig. 3, itself having formed from the merger of smaller subunits, and therefore illustrating the self-similar structure of the system. The ruler indicates a scale of $13.6 \mathrm{pc}$.

presented by Colín et al. (2013, hereafter C+13). This simulation utilized the N-body, AMR-Hydro code ART (Kravtsov et al. 1997) with (non-accreting) sink particles, adding a simplified treatment of radiative transfer that allows a first-order estimate for each sink's Strömgren radius. Cells within one Strömgren radius from a sink particle are heated to $T=10^{4} \mathrm{~K}$. Furthermore, a probabilistic SF scheme was used, which allowed the sink particles to take a power-law mass distribution, with a slope consistent with the Salpeter value. That is, in this simulation, the sink particles correspond to individual stars, with a realistic mass distribution. This allows following the cluster dynamics realistically as well. The box size is $256 \mathrm{pc}$, and the initial density and temperature in the box were $n=1 \mathrm{~cm}^{-3}$ and $T=5000 \mathrm{~K}$, respectively. Two colliding streams of gas, of radius $64 \mathrm{pc}$ and length $112 \mathrm{pc}$ and with opposite velocities of magnitude $5.9 \mathrm{~km} \mathrm{~s}^{-1}$ each, were set to collide at the $x=0$ plane in the simulation. A turbulent velocity field with a spectrum peaking at wavenumber $k=8 \times 2 \pi / L_{\text {box }}$ (so that the fluctuation size scale is smaller than the radius of the colliding streams) was applied to trigger instabilities in the compressed layer. For more details, we refer the reader to $\mathrm{C}+13$.

In this simulation, three main clusters form, two of which are sufficiently massive to disperse/evaporate the dense gas around them on a timescale of $\sim 13$ Myr. Stars begin to form at $t \approx 18.9 \mathrm{Myr}$, and the first HiI regions appear at $t \approx 24.2 \mathrm{Myr}$. At $t \approx 37.5$ Myr, the dense gas has been cleared from a radius of $\sim 70 \mathrm{pc}$ around the clusters. Figure 2 shows, in the left panel, one snapshot of the simulation, at $t=25.9$ Myr into the evolution, in which the growing HII regions around the two most massive clusters can be seen as faint shells around the clusters, while the filamentary structure of the cloud is still noticeable in general. The clusters themselves have formed along the main filamentary structures in the cloud, and contain $10^{3}-10^{4} \mathrm{M}_{\odot}$.

A crucial implication of the HGC scenario is that the filaments constitute part of the large-scale gravitational collapse, funneling gas into the cores within them, as observed by Gómez \& Vázquez-Semadeni (2014) in a numerical simulation of cloud formation and collapse. Moreover, these authors observed a hierarchy of collapses within the filaments, so that small clumps, which are sometimes forming stars already, are falling onto largerscale ones. This means that the late-stage clusters are assembled from smaller sub-units 

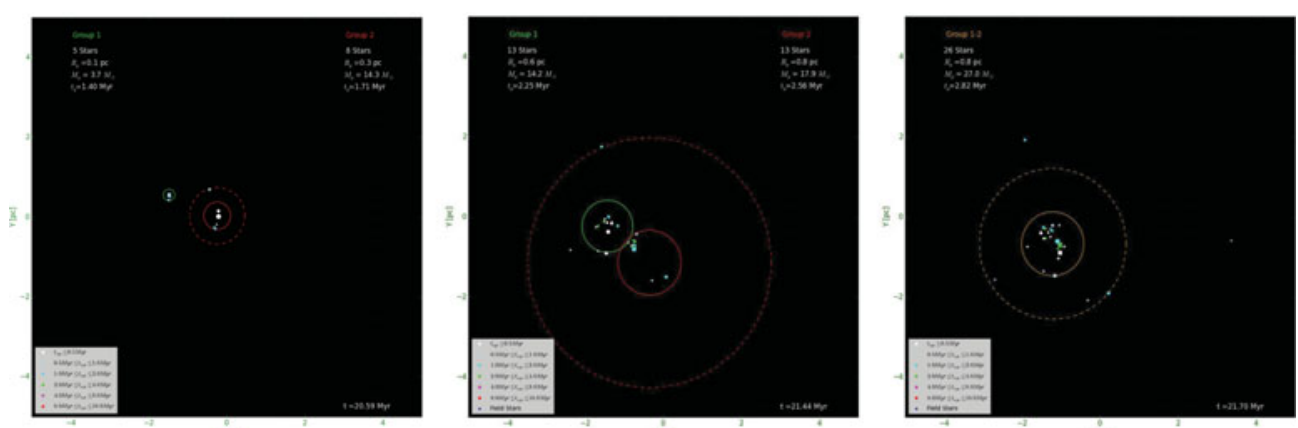

Figure 3. Three snapshots of "cluster 2" at times $t=20.59,21.44$, and 21.70 Myr in the simulation (respectively, left, middle, and right frames), showing the merging of two subunits to form the cluster.

that formed in the filaments in the periphery of the trough of the large-scale gravitational potential well. This is illustrated in the right panel of Fig. 2, which shows a zoom-in around the second most massive cluster (hereafter, "cluster 2") of those seen in the left panel, at $t=24.9 \mathrm{Myr}$. It can be seen that this cluster is actually composed of three groups at this time, which later (at $t \sim 30 \mathrm{Myr}$ ) merge to form a single larger cluster.

Operationally defining a group is a nontrivial task, because the gas clumps continue to form stars over extended periods of time due to the accretion from the filaments onto them. We thus define membership to a group by a sequence of steps. At early times, when the groups are clearly distinct by eye, we apply a "friends-of-friends" algorithm to the stars to define the groups. Subsequently, as new stars are formed, we assign them to the groups whose center of mass is closest to the new star. Also, we define the radius of the group at each time as the distance from its center of mass to the second most distant star. Finally, we say that two groups have merged when the distance between their centers of mass is smaller than the larger of the two radii.

The evolution and assembly of the central group in the right panel of Fig. 2 is illustrated in Fig. 3, which shows the projected positions of the sink particles on the $(x, y)$ plane at times $t=20.59,21.44$, and 21.70 Myr into the evolution of the simulation in the left, middle, and right panels, respectively. It is seen that, at the earlier time, the system consists of two subgroups, which approach each other and finally merge. In general, we observe this mechanism operating at all scales in our simulation, with groups in turn consisting of subgroups, and so on, implying that the clusters must exhibit a self-similar, fractal structure.

\section{Discussion}

The results described above suggest that the multi-scale, hierarchical gravitational collapse (HGC) of the clouds may be responsible for at least three observed properties of open clusters: 1) that there appears to be an increase of the SFR, so that the age distributions of clusters generally have a tail of older stars (3-7 Myr), while most stars are young (1-2 Myr; Palla \& Stahler 2000); 2) that they appear to have a self-similar structure, observationally manifested in an absence of a characteristic scale in the distribution of protostellar separations (Bressert et al. 2010). In our simulation, this is manifested in the hierarchical membership of the stellar units that conform a cluster; 3) that the clusters appear to consist of subunits of slightly different ages that merge to form larger structures (Kuhn et al. 2015b; Rivera-Gálvez et al. 2015). These are natural consequences of HGC in globally collapsing clouds. The global collapse produces an increase of the 
SFR. The hierarchical nature causes, at large scales, filamentary structures that feed the star-forming clumps for extended periods of time and, at small scales, it produces local star-forming sites that fall into the troughs of the large-scale contracting structures.

\section{References}

Bertoldi, F. \& McKee, C. F. 1992, ApJ, 395, 140

Bressert, E., Bastian, N., Gutermuth, R., et al. 2010, MNRAS, 409, L54

Colín, P., Vázquez-Semadeni, E., \& Gómez, G. C. 2013, MNRAS, 435, 1701

Federrath, C. \& Klessen, R. S. 2012, ApJ, 761, 156

Franco, J., Shore, S. N., \& Tenorio-Tagle, G. 1994, ApJ, 436, 795

Gao, Y. \& Solomon, P. M. 2004, ApJ, 606, 271

Gómez, G. C. \& Vázquez-Semadeni, E. 2014, ApJ, 791, 124

Hartmann, L., Ballesteros-Paredes, J., \& Heitsch, F. 2012, MNRAS, 420, 1457

Heitsch, F., Burkert, A., Hartmann, L. W., Slyz, A. D., \& Devriendt, J. E. G. 2005, ApJL, 633, L113

Hennebelle, P. \& Chabrier, G. 2011, ApJL, 743, L29

Hillenbrand, L. A. \& Hartmann, L. W. 1998, ApJ, 492, 540

Hoyle, F. 1953, ApJ, 118, 513

Kim, J. \& Ryu, D. 2005, ApJL, 630, L45

Kirk, H., Offner, S. S. R., \& Redmond, K. J. 2014, MNRAS, 439, 1765

Koyama, H. \& Inutsuka, S.-i. 2002, ApJL, 564, L97

Kravtsov, A. V., Klypin, A. A., \& Khokhlov, A. M. 1997, ApJS, 111, 73

Krumholz, M. R. \& McKee, C. F. 2005, ApJ, 630, 250

Kuhn, M. A., Getman, K. V., \& Feigelson, E. D. 2015a, ApJ, 802, 60

Kuhn, M. A., Feigelson, E. D., Getman, K. V., et al. 2015b, arXiv:1507.05653

Lada, C. J. \& Lada, E. A. 2003, ARA $\& A, 41,57$

Palla, F. \& Stahler, S. W. 2000, ApJ, 540, 255

Rivera-Gálvez, S., Román-Zúñiga, C. G., Jiménez-Bailón, E., et al. 2015, arXiv:1510.02835

Vázquez-Semadeni, E. 1994, ApJ, 423, 681

Vázquez-Semadeni, E., Gómez, G. C., Jappsen, A.-K., Ballesteros-Paredes, J., \& Klessen, R. S. 2009, ApJ, 707, 1023

Vishniac, E. T. 1994, ApJ, 428, 186

Williams, J. P. \& McKee, C. F. 1997, ApJ, 476, 166

Zamora-Avilés, M., Vázquez-Semadeni, E., \& Colín, P. 2012, ApJ, 751, 77

Zamora-Avilés, M. \& Vázquez-Semadeni, E. 2014, ApJ, 793, 84 